\title{
An international field study to investigate the psychometric properties of the updated EORTC QLQ-LC29 module for assessing quality of life in patients with lung cancer
}

Running head: Questionnaire for lung cancer patients

Michael Koller, Omar Shamieh, Marianne J Hjermstad, Kjersti Hornslien, Teresa Young, Tara Chalk, Georgios loannidis, Amelie Harle, Colin D Johnson, Krzysztof A Tomaszewski, Samantha Serpentini, Monica Pinto, Lotte van der Weijst, Annelies Janssens, Ofir Morag, Wei-Chu Chie, Juan I Arraras, Cecilia Pompili, Wolfgang Jungraithmayr, Marlene Hechtner, Despina Katsochi, Karolina Müller, Laura Gräfenstein, Christian Schulz, Andrew Bottomley on behalf of the EORTC Quality of Life Group, EORTC Lung Cancer Group, and European Society of Thoracic Surgeons

Center for Clinical Studies, University Hospital Regensburg, Regensburg, Germany (Prof M Koller PhD, K Müller MSc); Department of Palliative Care, King Hussein Cancer Center, Amman, Jordan (O Shamieh MD); Regional Advisory Unit for Palliative Care, Dept. of Oncology, Oslo University Hospital / European Palliative Care Research Centre (PRC), Dept. of Oncology, Oslo University Hospital, and Institute of Clinical Medicine, University of Oslo, Oslo, Norway (Prof M J Hjermstad PhD), Department of Oncology, Oslo University Hospital, Oslo- Norway (K Hornslien MD); East \& North Hertfordshire NHS Trust incorporating Mount Vernon Cancer Centre, Northwood, United Kingdom (T Young BSc., T Chalk); Oncology Department, Nicosia General Hospital, Nicosia, Cyprus (G loannidis MD); Poole Hospital NHS Foundation Trust, United Kingdom (A Harle MD); Surgical Unit, University of Southampton, Southampton, United Kingdom (Prof C D Johnson MChir); Faculty of Medicine and Health Sciences, Andrzej Frycz Modrzewski Krakow University, Krakow, Poland (Prof K A Tomaszewski, MD); Veneto Institute of Oncology IOV-IRCCS Comprehensive Cancer Center, Padova, Italy (S Serpentini PhD); Rehabilitation Medicine Unit, Department of Supportive Care, Istituto Nazionale Tumori - IRCCS- Fondazione G. Pascale, Naples, Italy (M Pinto MD); Department of Radiation Oncology, Ghent University Hospital, Ghent, Belgium ( $L$ van der Weijst MsC); Department of Thoracic Oncology, Antwerp University Hospital, Edegem, Belgium (A Janssens MD); Chaim Sheba Medical Center, Ramat-Gan, Israel (O Morag MD); Institute of Epidemiology and Preventive Medicine and Department of Public Health, College of Public Health, National Taiwan University, Taipei, Republic of Taiwan (Wei-Chu Chie MD PhD); Oncology Departments, Complejo Hospitalario de Navarra, Pamplona, Spain (J I Arraras PhD); Institute of Cancer and Pathology, University of Leeds, Leeds, United Kingdom (C Pompili MD PhD); Department of Thoracic Surgery, University Hospital Rostock, Rostock, Germany (Prof W Jungraithmayr MD PhD); University Medical Center, Johannes Gutenberg University Mainz, Mainz, Germany (M Hechtner PhD); Department of Radiation Oncology, Hygeia Hospital, Athens, Greece (D Katsochi MD); Department of Internal Medicine, University Hospital Regensburg, Regensburg, Germany (L Gräfenstein, Prof C Schulz MD); Quality of Life Department, EORTC, Brussels, Belgium (A Bottomley PhD)

Address for correspondence:

Prof. Dr. Michael Koller

Center for Clinical Studies

University Hospital Regensburg

D-93042 Regensburg

Germany

Phone : + 499419445630

Fax: $\quad+499419444462$

michael.koller@ukr.de 


\section{Summary}

\section{Background}

The EORTC QLQ-LC13 assesses quality of life (QoL) in patients with lung cancer (LC) and was the first EORTC module developed for use in international clinical trials. Since its publication in 1994, major treatment advances have occurred. This calls for an update of the module to improve the assessment and management of side effects, symptom burden, and quality of life. The paper presents results from the international psychometric validation study of the updated module.

\section{Methods}

This was an international, observational field study to investigate the psychometric properties of the updated LC-module. Psychometric analyses included confirmatory factor analysis and methods from classical test theory.

\section{Findings}

523 patients with confirmed diagnosis of lung cancer (either NSCLC or SCLC; 270 [51.6\%] NSCLC IV, 315 [60.2\%] male, Karnofksy Performance Status median 80 [IQR = 20]) from 19 centers in 12 countries participated. The updated module consists of 29 items, keeping 12 from the previous QLQ-LC13. Confirmatory factor analysis suggested five multi-item scales (Coughing, Shortness of breath, Fear of progression, Hair problems, Surgery-related symptoms ) and 15 single items: RMSEA $=0.075, \mathrm{GFI}=0.934, \mathrm{NFI}=0.877, \mathrm{CFI}=0.901$. Analyses of convergent and divergent validity confirmed this solution. Internal consistencies of all multi-item scales ranged between 0.73 and 0.86 . Test-retest reliabilities ranged between 0.82 and 0.97 . Four of the five multi-item scales yielded known group differences when patients with lower vs. higher Karnofsky Performance Status were contrasted ( $p<$ 0.007 ); so did 10 of the 15 single items. Three of the five multi-item scales showed responsiveness to change over time $(p<0.050)$; so did 9 out of 15 single symptoms.

\section{Interpretation}

The Phase 4 study determined the psychometric properties of the updated LC module, which is ready for use in international clinical lung cancer studies.

\section{Funding}

EORTC Quality of Life Group

KEY WORDS: quality of life, patient-reported outcomes (PRO), lung cancer, clinical trials, cross-cultural validation, EORTC QLQ-C30, EORTC QLQ-LC29 


\section{Research in context}

\section{Evidence before this study}

At the beginning (Phase 1) of this project to update the EORTC QLQ-LC13, a professional medical librarian performed an encompassing literature search for publications that related to the EORTC QLQ-LC13. The literature search covered the years from 01.01.1994 to 31.12.2013 and made use of a total of 36 databases, including the Cochrane Database of Systematic Reviews, the Cochrane Central Register of Controlled Trials, MEDLINE, EMBASE, PSYNDEX, PsycINFO, Social SciSearch, and the Health Technology Assessment Database. The syntax contained the term "LC13" in any combination with the terms EORTC, quality of life (questionnaire), lung, and module. This search was supplemented by a hand search.

At total of 240 studies were included in the analysis, of which $109(45.2 \%)$ were randomized controlled clinical trials (RCTs). This literature review proved the frequent utilization and acceptance of the QLQ-LC13.

In addition, interviews with patients and health care professionals were conducted to provide a comprehensive list of issues that are relevant with respect to the quality of life of patients with lung cancer. It became apparent that numerous side effect issues were missing that are relevant with regard to newly available therapeutic options. Phase 2 of the project provided an amended provisional questionnaire, and Phase 3 comprised an international study to investigate comprehensibility and acceptance of this provisional questionnaire.

\section{Added value of this study}

The Phase 4 study determined the psychometric properties of the updated lung cancer module, in terms of reliability, validity and responsiveness to change. The updated module contains a total of 29 items. It retained 12 of the 13 original QLQ-LC13 items and was supplemented with new items that assess side effects of targeted therapy, immunotherapy, radio-chemotherapy, and surgery. It is composed of five multi-item scales (coughing, shortness of breath, hair problems, fear of progression, surgical symptoms) and 15 single items. 24 items should be administered to lung cancer patients in a standard fashion; the five-item surgical sub-scale is optional and is only applicable for patients who have had thoracic surgery (24 plus 5 item module concept).

\section{Implications of all the available evidence}

The EORTC QLQ-LC29 promises to be the new standard for QoL assessment in patients with lung cancer. It is available in numerous validated translations and is ready to be used in international clinical lung cancer trials. 


\section{Introduction}

According to the GLOBOCAN analysis on the worldwide cancer incidence, lung cancer is the most commonly diagnosed malignancy (1.82 million) that also accounted for the highest number of cancer deaths (1.6 million deaths) ${ }^{1}$.

Smoking is widely acknowledged as the leading cause of lung cancer ${ }^{2}$. In addition, genetics, pollution and occupational exposure, socioeconomic factors, and gender play a role in the epidemiology of lung cancer.

Quite generally, lung cancer has a poor prognosis, although the 5-year relative survival rates for all types of lung cancer increased from $10 \cdot 7 \%$ to $19 \cdot 8 \%$ in the past four decades ${ }^{3}$.

This progress can be attributed to improved standards including early detection, molecular characterization, staging, surgery, radiotherapy, and systemic therapies including targeted therapy and immunotherapy 4,5

These treatments, often combined in a multimodality approach ${ }^{6}$, not only promise to improve survival, but may affect patients' quality of life (QoL). QoL refers to patients' subjective experience of their illness in the somatic, psychological and social domains. Clearly, QoL may be impaired by the disabling disease itself, but also by side-effects of the therapy. Thus, an overall assessment of a therapy can only be made when traditional clinical endpoints are considered in combination patients' perception of the illness and therapy. Hence, the availability of valid measures for patient-reported outcomes (PROs) such as QoL are of the utmost importance. Such measures need to be clinically meaningful and methodologically sound.

A standard instrument that fulfills these criteria and hence has been used in hundreds of lung cancer trials is the EORTC QLQ-LC13 ${ }^{7,8}$. This module has been developed for use alongside the core questionnaire EORTC QLQ-C30 ${ }^{9}$, was published in $1994{ }^{10}$ and has been widely used in clinical trials

Given that the new therapeutic options bring along new efficacy measures and novel side effects that are different from those associated with classic anticancer treatments, the EORTC initiated a research project to update the QLQ-LC13. The first three phases of the research project have led to a new lung cancer module QLQ-LC29, which preserved 12 of the 13 original items, and added items on relevant and common side effects and a surgical subscale (Table 1). The new module proved to be acceptable and comprehensible and was perceived as highly relevant by lung cancer patients in an international Phase 3 study ${ }^{11}$.

The present paper reports the international Phase 4 field study, which was designed to investigate the scale structure and the psychometric properties of the QLQ-LC29. 


\section{Methods}

\section{Overview}

The EORTC Quality of Life Group has implemented a systematic, stepwise methodology to develop modules ${ }^{12}$. Results from phases 1 to 3 are summarized in Appendix $p 1{ }^{10,11}$. The present paper reports the final Phase 4 of the project, the international validation of the module. For this purpose, international translations of the questionnaire according to the EORTC translation guidelines were available ${ }^{13}$.

Approval was obtained from the Ethics Committee of the University of Regensburg (16 March 2016, reference number 16-101-0059). In addition, the study protocol was approved by local ethical committees of participating centers according to the national requirements. The study was registered with clinicaltrials.gov (ClinicalTrials.gov, Identifier: NCT02745691).

\section{Patient eligibility criteria}

Patient were eligible for this study if they had a confirmed diagnosis of lung cancer (either based on histology, cytology and/or pathology), no previous other primary tumor, were mentally fit with sufficient language skills to understand and complete the questionnaire, were above 18 years of age, and gave written informed consent to participate in the study.

\section{Procedure}

Local investigators informed patients about the purpose of the study and obtained signed informed consent during a hospital visit. Patients were asked to fill in the paper versions of the core questionnaire EORTC QLQ-C30 plus QLQ-LC29 on their own, but received assistance upon request. Time frames for QoL assessment were specified in relation to the primary therapy patients underwent at the time of study enrollment (Appendix $p 2$ ). Time frames were chosen so that the module would be able to tap into therapy-related side effects.

Investigators used a debriefing interview form to record patients' perception of the questionnaire (time of completion, need for help, confusing or upsetting questions, or comments about the questionnaire). The responsible investigators completed a case report form to document disease and therapy-related information.

Furthermore, investigators were required to select approximately half of their patients to fill in the questionnaire at a second time-point two to four weeks later, either in hospital or via regular mail. These patients should either appear to be clinically stable (rendering appropriate for assessing test-retest reliability) or prone to changes in their well-being due to side-effects or due to the palliative effect of the therapy. Patients who filled in the questionnaire at a second time point responded to an anchor question to indicate if they had experienced changes in their health and symptoms between the two assessment points (better, unchanged, worse).

\section{Sample size considerations}

The primary aims of the study are to evaluate the factor structure and psychometric properties of the EORTC QLQ-LC29 in patients with lung cancer. Sample size is determined by the number of items in the questionnaire. The LC29 contains 24 items that apply to all lung cancer patients and additional 5 items that only apply to surgical patients. According to 
the 'rule of thumb', $10-15$ cases per item are needed ${ }^{14}$. Therefore, the required number of patients ranges between 290 and 435. A further aspect that needs to be considered is the distribution across response options. It has been argued that at least $5 \%$ responses in each category are required to obtain stable parameter estimation ${ }^{15}$. Thus, we decided to include a minimum of 450 patients for the main psychometric analyses, and this minimum number was allowed to inflate to compensate for missing responses.

\section{Statistical analyses}

The primary endpoints were the assessments of the scale structure and psychometric properties including reliability, sensitivity and responsiveness to change of the updated EORTC QLQ-LC29.

Scale structure was analysed using confirmatory factor analysis (CFA), exploratory factor analyses (EFA) as well as convergent and discriminant validity. CFA was used to verify the hypothesized factor structure of the variable set. Fit indices determine that appropriateness of a tested model. Factor loadings refer to item/scale correlations, whereby a factor loading should be $\geq 0.40$ to indicate a sufficient correlation.

Based on results of Phase 3, it was hypothesized that the QLQ-LC29 includes five multi-item scales (coughing, shortness of breath, tumor progression, side effects, and surgical symptoms) and five single items (cough up blood, pain in chest/arm or shoulder/other parts of your body, weight loss). As the CFA did not confirm the hypothesized factor structure, EFA were conducted to identify the underlying structure of variables. To verify the new factor structure from EFA, CFA as well as convergent and discriminant validity were calculated. Convergent validity is based on item-own scale correlations (corrected for overlap) and discriminant validity is based on item-other scale correlations. A definite scaling error existed if an item correlates significantly less to its own scale than to another scale. Thus, scaling errors were determined by comparing the correlation coefficients. Items showing a definite scaling error should be excluded from a scale.

Reliability was calculated by means of Cronbach's alpha (internal consistency) and intraclass coefficient (ICC, test-retest reliability).

Sensitivity of the module was assessed by means of known group differences according to the Karnofsky Performance Status (independent t-tests).

Responsiveness to change over time was calculated using the differences between the second and first assessment and patients' responses to an anchor question ("relative to my first assessment my symptoms got better/did not change/got worse). The three groups of patients were compared using ANOVA.

In addition, all scores for the EORTC QLQ-C30 were calculated and known group differences and responsiveness to change were reported.

All tests were two-tailed with a significance level of $p<0 \cdot 05$. Descriptive statistics included counts $(n)$, percentages (\%), means $(m)$, medians (med) and interquartile range (IQR). Occassionaly percentages may not add up to $100 \%$ due to missing responses.

SAS 9.4 (SAS Institute Inc., Cary, NC, USA) was used to calculate CFA, and IBM SPSS Statistics 25.0 (IBM Corporation, Armonk, NY, USA) was the statistical analysis tool for all other analyses. 


\section{Role of the funding source}

The funder of the study had no role in the collection, analysis, or interpretation of the data, nor in the writing of the report or research paper. The corresponding author had full access to all of the data and the final responsibility to submit the manuscript for publication. 


\section{Results}

Based on the results of the Phase 3 study, we started with the hypothesis that the QLQ-LC29 is composed of five multi-item scales (coughing, shortness of breath, tumor progression, side effects, and surgical symptoms) and five single items (cough up blood, pain in chest/arm or shoulder/other parts of your body, weight loss). First, we conducted a CFA to verify whether the hypothesized factor structure could be supported. CFA yielded poor fit for this model. Table 1 (original model) presents results of CFA and thresholds for acceptable and good fit of the fit indices which show how well the hypothesized factors were measured ${ }^{16}$. The following indices were used: Chi-Square $\left(X^{2}\right)$, root mean square error of approximation (RMSEA), global goodness-of-fit-index (GFI), adjusted goodness-of-fit-index (AGFI), normedfit index (NFI), Tucker-Lewis index (TLI), and comparative-fit index (CFI).

We then computed EFA to find an appropriate factor structure. EFA supported the factors coughing, shortness of breath, tumor progression, and surgical symptoms. The items of the hypothesized side effect factor and single items were grouped in four additional factors. The only factor that was clinically interpretable and had a sufficiently high Cronbach's alpha was a dimension related to hair, composed of item nos. 39 (hair loss) and 50 (thin hair).

We then repeated CFA based on the findings of the EFA, hypothesizing the factors coughing, shortness of breath, tumor progression, surgical symptoms, hair problems, and an overall side effect/symptom factor. The fit parameters slightly improved but were still not satisfactory (Table 1 adapted model). In particular, nine out of the 15 items had standardized factor loadings (relationship between variable and underlying factor) $<0.40$ (ranging between 0.17 and 0.36 ). We therefore decided to treat all 15 side effect/symptoms as single items rather than as a coherent factor. This model yielded acceptable to good values for four out of six fit indicators (Table 1 final model). All factor loadings of the individual items were above the threshold of 0.40 , and 23 of the 29 factor loadings were $>0.70$ (Table 2).

We also conducted analyses according to classical test theory. The criterion of convergent validity was set at $>0.40$ (corrected for overlap). The factors coughing, shortness of breath, tumor progression, surgical symptoms, and hair clearly met this criterion (Table 3 ). The correlation pattern of the coefficients of the symptoms/side effects factor supported the decision to treat the 15 symptoms as single items rather than as a coherent factor. The criterion of discriminant validity was set at $<0 \cdot 40$. Even though some correlations between an item and another scale were above $0 \cdot 40$, no definitive scaling error occurred. Thus, the revised scale structure of the EORTC QLQ-LC29 was supported.

As can be seen in Table 2, internal consistency of the proposed multi-item scales coughing, shortness of breath, tumor progression, hair problems, and surgical symptoms were above the commonly accepted threshold of $0.70{ }^{17}$, with Cronbach's alphas ranging between 0.73 and $0 \cdot 86$. Furthermore, the 15 single symptoms/side effects items added up to scale with acceptable Cronbach's alpha $=0 \cdot 71$.

Altogether, 195 (37.3\%) of 523 patients filled in a second questionnaire (median follow-up time $=14$ days, IQR $=15.3$ days) and $81(41.5 \%)$ of these 195 patients reported that they had experienced no change in their symptoms between the first and second assessment. Thus, these patients were used for computing test-retest reliability by means of the Intraclass-Coefficient (ICC, Table 2). ICC values were high, ranging between 0.82 and 0.97. Even ICCs for single items were $<0.70$ in five out 15 items. The symptom/side effect burden score had an ICC $=0.89$. 
At the second assessment point, patients indicated whether their symptoms got worse, got better, or whether there was no change. For each QoL scale we computed pre-post change scores and compared the three groups using one-way ANOVA. There should be no pre-post difference in the "no change" group, a change to the positive in the "got better" group, and a change to the negative in the "got worse" group. A significant one-way ANOVA would indicate responsiveness to change for a given scale. Three out of the five QLC-LC29 scales (shortness of breath, fear of progression, hair problems) showed group differences, as did the symptom/side effect burden score (Table 4). In addition, nine out of the 15 single items evidenced responsiveness to change.

Physicians' assessment of the Karnofsky Performance Status was taken as an indicator of patients' overall health at the time of the first QoL assessment. Based on median split, two groups of patients were compared, those with relatively good health $(\geq 80)$ and those with poorer health $(\leq 70)$. As to be seen in Table 5, four out of the five QLQ-LC29 scores (coughing, shortness of breath, fear of progression, surgery-related symptoms) showed significant mean differences in the expected directions (ps $<0.0001$ ), as did 10 out of the 15 single symptoms ( $p s<0.050)$.

Furthermore, all scores of the QLQ-C30 evidenced known-group differences in the expected direction.

Appendix $\mathrm{p} 3$ shows the means of the 15 single symptoms as well as their summary score across the treatments that patients have received at the time of their QoL assessment. The two highest means per symptom are displayed in bold. As can be seen, there is a variation of single symptoms across treatments, and statistically significant differences were found for 12 out of the 15 symptoms. Furthermore, the overall symptom/side effect burden score was statistically significant. The overall score was most pronounced for patients undergoing radiotherapy and patients undergoing targeted therapy.

Between April 12, 2016 and September 26, 2018, 523 patients in 19 centers from 12 countries, representing English-speaking, Northern European, Southern European, Eastern European, and non-European regions were recruited for this international multicenter study (Table 6). Median age was 66 years (IQR = 14 years), and the majority were male (315 [60.2\%] out of 523 patients) (Table 6). Most patients had advanced disease (NSCLC IV, 270 [51.6\%] out of 523 patients) and received treatment with palliative intent (351 [67.1\%] out of 523 patients). The median time from diagnosis to study enrollment was six months (IQR = $16 \cdot 2)$. More than half of the patients $(279$ [53.3\%] of 523) received more than one therapy in the course of their patient career. Patients were enrolled and characterized according to their ongoing therapy at the time of the QoL assessment (Appendix $\mathrm{p} 2$ ).

More than half of patients (277 [53\%] of 523 patients) spent less than 10 minutes completing the questionnaire, while $23(4.4 \%)$ out of 523 patients needed 21 minutes or longer. Two thirds of the patients (334 [63.9\%] out of 523 patients) filled in the questionnaire themselves. In cases where assistance was needed (168 [32.1\%] out of 523$)$, this was provided by a family member or a member of the research team and consisted of explaining/clarifying questions or reading them out.

$80(15.3 \%)$ out of 523 patients found some questions difficult to understand. These included items related to hair loss (item no. 39), thin hair (item no. 50), decrease in physical capabilities (item no. 53), and weight loss (item no. 54). Only $15(2.9 \%)$ out of 523 patients found questions upsetting. These included items related to tumor progression and future 
health (items no. 49 and 51), but also financial difficulties (item no. 28), and being reminded about health problems quite generally.

Overall, compliance was high, with a rate of missing items as low as $237(0 \cdot 8 \%)$ out of 28005 reponses to the QLQ-C30 and QLQ-LC29 (exluding the surgical items) in the first assessment, and $107(1.0 \%)$ out of 10530 in the second assessment. The items with the highest numbers of missing responses (8 [1.5\%] out of 523 ) were three items of the QLQC30 (depressed, difficulty remembering, financial difficulties), and two items of the QLQLC29 (shortness of breath when climbing stairs, thin hair). 


\section{Discussion}

The scale structure of the updated module to assess quality of life in lung cancer patients EORTC QLQ-LC29 was best compatible with a model that is composed of five multi-item scales (Coughing, Shortness of breath, Fear of progression, Hair problems, Surgery-related symptoms) and 15 single items (e.g., Tingling hands or feet or Dizziness). This solution is supported by CFA. Four out of the six goodness-of-fit-indices showed acceptable to good values ${ }^{16}$. The chi-square test was significant in all three models, but the appropriateness of this statistic is under dispute ${ }^{18,19}$, whereas the other fit indices are recommended. Importantly and in line with CFA requirements, the factor loadings of the individual items exceed the $>0.40$ threshold. The proposed CFA solution may not be perfect, but is backed by classical test theory in terms of convergent and divergent validity and lack of scaling error.

The initial model hypothesized a 12-item side effect scale (in addition to four multi-item scales and five single items), and the adapted model hypothesized a 15 -item side effect scale. None of these models were supported by CFA. The critical component was the symptom/side effects factor and reflects the fact that symptoms may vary across diagnoses, disease states and course of therapies. Hence their clustering in a syndrome may be dependent on a patient's actual situation ${ }^{20}$. As Appendix $\mathrm{p} 3$ illustrates, single symptoms varied according to the type of therapy that the patients received at the time of study. For instance, mouth soreness was most pronounced in patients undergoing radio-chemotherapy or targeted therapies, decrease in physical capabilities was rated highest in patients with relatively recent surgery, and skin problems were most prevalent in patients undergoing targeted therapies or immunotherapy.

It is also interesting to note that the original lung cancer module also contained a high number of single items, namely 10 single items out of a total of 13 items. Also other EORTC modules have relatively high numbers of single items, e.g. the colorectal cancer module QLQ-CR29 with 19 single items ${ }^{21}$. Nevertheless, for informative purposes it may be useful to sum up the 15 single items in the sense of an overall lung cancer symptom/side effects burden score, which also showed an acceptable internal consistency according to Cronbach's alpha.

All five multi-item scales had acceptable to very good internal consistency, and good to excellent test-retest reliability (Table 2 ).

The new QLQ-LC29 also proved to be sensitive. With regard to known-group differences, four out of the five multi-item sales discriminated between patients with high versus low scores on the Karnofsky Performance Status; so did ten out of the 15 single symptoms, as well as the symptom/side effect burden score (Table 5). We also observed responsiveness to change: three out of the five multi-item scales (shortness of breath, fear of progression, hair problems) discriminated between patients who indicated that their health improved, got worse or remained unchanged between the two QoL assessment points. Further, nine of the 15 single symptoms as well as the symptom/side effect burden score were responsive to change.

It should be noted that most of the analyses discussed above were also conducted for the core questionnaire QLQ-C30. As has been shown so often previously, the QLQ-C30 proved to be reliable, cross-culturally valid as well as sensitive in terms of known-group differences and responsiveness to change. Importantly, this shows that the QLQ-C30 contains a number of symptoms that are relevant also for innovative therapies (for instance, diarrhea as side 
effect of immunotherapy). Therefore, the new lung cancer module QLQ-LC29 should always be used in conjunction with the QLQ-C30.

Patients found the questionnaire highly acceptable and the number of missing responses to questionnaire items was low. From a user perspective, the QLQ-LC29 displays three qualities at the same time: traditional, innovative and flexible. "Traditional" in the sense that 12 out of the 13 items were retained in the new module, which underscores the relevance of the previous QLQ-LC13. Thus Items 31 to 42 were taken from the original questionnaire, whereas items 43 to 59 were newly added (Appendix $p$ 4). This allows for comparability of data from studies that employed the original QLQ-LC13. Only the item "Did you take any medicine for pain" was removed after Phase 3 because of its low relevance ratings and notoriously high number of missing responses.

The QLQ-LC29 is innovative in the sense that the module contains new symptom items, such as splitting fingernails and burning eyes that specifically address toxicity of novel systemic therapies, such as EGFR-targeted agents The module also contains a 5 -item scale specifically designed to address issues of patients who had undergone surgery for lung cancer (e.g., pain in the area of surgery, sensitivity of the wound). It enables thoracic surgeons to assess the outcome of surgical management and its relation to multi-modal treatment ${ }^{22}$.

The QLQ-LC29 is flexible because the surgery-scale may be omitted in studies that involve patients who have never undergone thoracic surgery for lung cancer. Removing the surgeryscale does not lead to loss of information or interference with other scales because it is the final scale on the questionnaire. Thus, the QLQ-LC29 should be regarded as a QLQ-LC24 plus 5 module.

EORTC is now employing a dynamic test strategy, in the sense that additional items from an encompassing item library to may be added to the core questionnaire and/or to a module $e^{23}$. This permits evaluation of specific, relevant issues that may be relevance in a given context, when they are not included in the established module of choice. This tailored strategy helps to even better capture the patient perspective. ${ }^{23}$

The QLQ-LC29 is also competitive in relation to other currently available measures for lung cancer ${ }^{24-28}$. The new module has been developed according to a rigorous state-of-the methodology in a cross-cultural setting ${ }^{12}$. The contents of the updated module reflect side effects that may come along with newly available therapeutic options for lung cancer. Very importantly, patients were an integral part in the entire development process with more than 800 patients being involved throughout Phases 1 to and 4 to shape the scope of the new module ${ }^{11}$. Thus, patients were given a strong voice, which fulfills an important methodological criterion of questionnaire development demanded by regulatory bodies 29,30 .

Limitations of the study relate to the less-than perfect CFA results and the relatively low number of patients involved in responsiveness of change analyses. Since questionnaire validation can be thought of as an ongoing process, further data are welcome to amend the present findings. Furthermore, the present study was not designed to conduct cross-cultural comparisons. This should be a topic for future trials. Most importantly, however, further large scale RCTs are needed that show how the module performs in the context of clinical trials.

In conclusion, the QLQ-LC29 retained 12 of the 13 original QLQ-LC13 items and contains new items that assess therapy side effects of targeted therapy, immunotherapy, radio- 
chemotherapy, as well as thoracic surgery. The Phase 4 study proved the psychometric properties and cross-cultural validity of the updated lung cancer module. The QLQ-LC29 is available in numerous validated translations and is ready to be used in international clinical lung cancer trials.

\section{Contributors}

OS, KH, TY, GI, AH, CDJ, KAT, SaSe, MP, LW, AJ, WC, JIA, CP, WJ, MH, DK, LG, and CS recruited patients, commented on previous versions of the manuscript, and approved the final manuscript. In addition, MHJ helped finalizing the study protocol. TC recruited patients and commented on previous versions of the manuscript. OM recruited patients and approved the final manuscript. AB helped in designing the study and interpreting the study results, commented on previous versions of the manuscript, and approved the final manuscript. KM and MK were responsible for the statistical analyses. MK designed the study, was responsible for the conduct of the study and drafted the first version of the paper.

We thank Monika Schöll for her language expertise in editing the manuscript.

\section{Declaration of interest}

MK reports grants from EORTC to conduct this study; outside the submitted work he received personal fees from Janssen-Cilag, Lilly and MSD outside. MH joined Boehringer Ingelheim after she finished recruiting patients to this study. MP reports personal fees from AMGEN ING, USA, and case payments for recruiting patients to this study. TY, TC, GI, KT, $\mathrm{LW}$, and JA report case payments for recruiting patients to this study. All other authors report no competing interests.

\section{Acknowledgements}

The work reported in this manuscript was supported by a grant by the European Organisation for Research and Treatment of Cancer to the first author (Koller Lung 03/2016), and the manuscript was approved by the Executive Committee of the EORTC Quality of Life Group. We thank Lana Abusalem for her help in recruiting patients. We are grateful to Ms Monika Schöll for her linguistic expertise.

\section{Data sharing}

Data will be shared according to the EORTC data release policy (https://www.eortc.org/datasharing/). 


\section{References}

1 Ferlay J, Soerjomataram I, Dikshit R, et al. Cancer incidence and mortality worldwide: Sources, methods and major patterns in GLOBOCAN 2012. Int J Cancer 2015; 136(5): E359-86 [https://doi.org/10.1002/ijc.29210][PMID: 25220842]

2 Islami F, Torre LA, Jemal A. Global trends of lung cancer mortality and smoking prevalence. Transl Lung Cancer Res 2015; 4(4): 327-38 [https://doi.org/10.3978/j.issn.2218-6751.2015.08.04][PMID: 26380174]

3 Lu T, Yang X, Huang Y, et al. Trends in the incidence, treatment, and survival of patients with lung cancer in the last four decades. Cancer Manag Res 2019; 11: 943-53 [https://doi.org/10.2147/CMAR.S187317][PMID: 30718965]

4 Besse B, Adjei A, Baas P, et al. 2nd ESMO Consensus Conference on Lung Cancer: Non-small-cell lung cancer first-line/second and further lines of treatment in advanced disease. Ann Oncol 2014; 25(8): 1475-84 [https://doi.org/10.1093/annonc/mdu123][PMID: 24669016]

5 Tan W-L, Jain A, Takano A, et al. Novel therapeutic targets on the horizon for lung cancer. The Lancet Oncology 2016; 17(8): e347-e362 [https://doi.org/10.1016/S1470-2045(16)30123-1]

6 Jones CM, Brunelli A, Callister ME, Franks KN. Multimodality Treatment of Advanced Non-small Cell Lung Cancer: Where are we with the Evidence? Curr Surg Rep 2018; 6(2): 5 [https://doi.org/10.1007/s40137-018-0202-0][PMID: 29456881]

$7 \quad$ Koller M, Warncke S, Hjermstad MJ, et al. Use of the lung cancer-specific Quality of Life Questionnaire EORTC QLQ-LC13 in clinical trials: A systematic review of the literature 20 years after its development. Cancer 2015; 121(24): 4300-23

[https://doi.org/10.1002/cncr.29682][PMID: 26451520]

8 Reale ML, Luca E de, Lombardi P, et al. Quality of life analysis in lung cancer: A systematic review of phase III trials published between 2012 and 2018. Lung Cancer 2020; 139: 47-54

[https://doi.org/10.1016/j.lungcan.2019.10.022.][PMID: 31734586]

9 Aaronson NK, Ahmedzai S, Bergman B, et al. The European Organization for Research and Treatment of Cancer QLQ-C30: A quality-of-life instrument for use in international clinical trials in oncology. J Natl Cancer Inst 1993; 85(5): 365-76 [https://doi.org/10.1093/jnci/85.5.365][PMID: 8433390]

10 Bergman B, Aaronson NK, Ahmedzai S, Kaasa S, Sullivan M. The EORTC QLQLC13: a modular supplement to the EORTC core quality of life questionnaire (QLQ-C30) for use in lung cancer clinical trials. European Journal of Cancer 1994; 30(5): 635-42 [https://doi.org/10.1016/0959-8049(94)90535-5]

11 Koller M, Hjermstad MJ, Tomaszewski KA, et al. An international study to revise the EORTC questionnaire for assessing quality of life in lung cancer patients. Ann Oncol 2017; 28(11): 2874-81

[https://doi.org/10.1093/annonc/mdx453][PMID: 28945875] 
12 Johnson CD, Aaronson NK, Blazeby JM, et al. EORTC quality of life group guidelines for developing questionnaire modules. $4^{\text {th }}$ ed. Brussels: EORTC 2011.

13 Kulis D, Bottomley A, Velikova G, Greimel E, Koller M, on behalf of the EORTC Quality of Life Group. EORTC Quality of Life Group: Translation Procedure; 2017 [cited 2020 January 8] Available from: URL: https://qol.eortc.org/manual/translation-procedure/.

14 Tabachnick BG, Fidell LS. Using multivariate statistics. 4th ed. Boston, MA: Allyn and Bacon 2001.

15 Petersen MA, Groenvold M, Aaronson NK, et al. Development of computerized adaptive testing (CAT) for the EORTC QLQ-C30 physical functioning dimension. Qual Life Res 2011; 20(4): 479-90

[https://doi.org/10.1007/s11136-010-9770-x][PMID: 20972628]

16 Simon D, Kriston L, Loh A, et al. Confirmatory factor analysis and recommendations for improvement of the Autonomy-Preference-Index (API). Health Expect 2010; 13(3): 23443

[https://doi.org/10.1111/j.1369-7625.2009.00584.x][PMID: 20579122]

17 Nunnally JC, Bernstein IH. Psychometric theory. 3rd ed. New York: McGraw-Hill 1994.

18 Fayers PM, Machin D. Quality of life: The assessment, analysis, and reporting of patient-reported outcomes. 3rd ed. Chichester, West Sussex, UK, Hoboken, NJ: John Wiley \& Sons Inc 2016.

19 Gundy CM, Fayers PM, Groenvold M, et al. Comparing higher order models for the EORTC QLQ-C30. Qual Life Res 2012; 21(9): 1607-17

[https://doi.org/10.1007/s11136-011-0082-6][PMID: 22187352]

20 Kristensen A, Solheim TS, Amundsen T, et al. Measurement of health-related quality of life during chemotherapy - the importance of timing. Acta Oncol 2017; 56(5): 737-45 [https://doi.org/10.1080/0284186X.2017.1279748][PMID: 28117614]

21 Whistance RN, Conroy T, Chie W, et al. Clinical and psychometric validation of the EORTC QLQ-CR29 questionnaire module to assess health-related quality of life in patients with colorectal cancer. Eur J Cancer 2009; 45(17): 3017-26

[https://doi.org/10.1016/j.ejca.2009.08.014][PMID: 19765978]

22 Pompili C. Quality of life after lung resection for lung cancer. J Thorac Dis 2015; 7(Suppl 2): S138-44

[https://doi.org/10.3978/j.issn.2072-1439.2015.04.40][PMID: 25984359]

23 Kuliś D, Piccinin C, Bottomley A, Groenvold M. EORTC Quality of Life Group Item Library: Technical Guidelines; 2018 [cited 2019 June 17] Available from: URL: https://www.eortc.org/app/uploads/sites/2/2018/09/IL-manual-20180305.pdf.

24 Hollen PJ, Gralla RJ, Kris MG, et al. Measurement of quality of life in patients with lung cancer in multicenter trials of new therapies. Psychometric assessment of the Lung Cancer Symptom Scale. Cancer 1994; 73(8): 2087-98

[PMID: 8156514] 
25 Cella DF, Bonomi AE, Lloyd SR, Tulsky DS, Kaplan E, Bonomi P. Reliability and validity of the Functional Assessment of Cancer Therapy-Lung (FACT-L) quality of life instrument. Lung Cancer 1995; 12(3): 199-220

[PMID: 7655830]

26 McCarrier KP, Atkinson TM, DeBusk KPA, Liepa AM, Scanlon M, Coons SJ.

Qualitative Development and Content Validity of the Non-small Cell Lung Cancer Symptom Assessment Questionnaire (NSCLC-SAQ), A Patient-reported Outcome Instrument. Clin Ther 2016; 38(4): 794-810

[https://doi.org/10.1016/j.clinthera.2016.03.012.][PMID: 27041408]

27 Tan J-Y, Yorke J, Harle A, et al. Assessment of Breathlessness in Lung Cancer:

Psychometric Properties of the Dyspnea-12 Questionnaire. J Pain Symptom Manage 2017; 53(2): 208-15

[https://doi.org/10.1016/j.jpainsymman.2016.08.009][PMID: 27720789]

28 Edbrooke L, Denehy L, Parry SM, Astin R, Jack S, Granger CL. How is physical activity measured in lung cancer? A systematic review of outcome measures and their psychometric properties. Respirology 2017; 22(2): 263-77 [https://doi.org/10.1111/resp.12975][PMID: 28102971]

29 Kluetz PG, Chingos DT, Basch EM, Mitchell SA. Patient-Reported Outcomes in Cancer Clinical Trials: Measuring Symptomatic Adverse Events With the National Cancer Institute's Patient-Reported Outcomes Version of the Common Terminology Criteria for Adverse Events (PRO-CTCAE). Am Soc Clin Oncol Educ Book 2016; 35: 67-73 [https://doi.org/10.14694/EDBK_159514.][PMID: 27249687]

30 Basu Roy U, King-Kallimanis BL, Kluetz PG, Selig W, Ferris A. Learning from Patients: Reflections on Use of Patient-Reported Outcomes in Lung Cancer Trials. J Thorac Oncol 2018; 13(12): 1815-7

[https://doi.org/10.1016/j.jtho.2018.09.003][PMID: 30337169] 


\section{Tables}

Table 1 Confirmatory factor analysis: Global fit for all models tested

Table 2 Internal consistency, Test-retest reliability, standardized factor loadings

Table 3 Convergent and discriminant validity

Table 4 Known group differences: Karnofsky Performance Status

Table 5 Responsiveness to change

Table $6 \quad$ Patient characteristics $(\mathrm{N}=532)$ 
Table 1 Confirmatory factor analysis: Global fit for all models tested

\begin{tabular}{|c|c|c|c|c|c|c|c|c|c|c|}
\hline$N=523$ & $x^{2}$ & df & $p$ & $X^{2} / d f$ & RMSEA & GFI & AGFI & NFI & TLI & CFI \\
\hline Tresholds for acceptable fit ${ }^{21}$ & & & $\geq 0.050$ & $\leq 5.00$ & $\leq 0.080$ & $\geq 0.800$ & $\geq 0.850$ & $\geq 0.800$ & $\geq 0.800$ & $\geq 0.850$ \\
\hline Tresholds for good fit ${ }^{21}$ & & & $\geq 0.010$ & $\leq 3.00$ & $\leq 0.050$ & $\geq 0.900$ & $\geq 0.950$ & $\geq 0.950$ & $\geq 0.950$ & $\geq 0.950$ \\
\hline Original Model & $1098 \cdot 790$ & 225 & $<0.0001$ & $4 \cdot 88$ & 0.090 & 0.828 & 0.771 & 0.634 & 0.607 & 0.680 \\
\hline Adapted Model & $825 \cdot 549$ & 247 & $<0.0001$ & 3.34 & 0.070 & 0.860 & 0.830 & 0.725 & 0.763 & $0 \cdot 788$ \\
\hline Final Model & $370 \cdot 233$ & 100 & $<0.0001$ & 3.70 & 0.075 & 0.934 & 0.801 & 0.877 & 0.726 & 0.901 \\
\hline
\end{tabular}

$\chi^{2}=$ Chi-Square, $\mathrm{df}=$ degrees of freedom, RMSEA $=$ root mean square error of approximation, GFI $=$ global goodness-of-fit-index, AGFI $=$ adjusted goodness-of-fit-index, NFI $=$ normed-fit index, TLI $=$ Tucker-Lewis index, $\mathrm{CFI}=$ comparative-fit index 
Table 2 Internal consistency, Test-retest reliability, standardized factor loadings

\begin{tabular}{|c|c|c|c|c|}
\hline Scale & Item \# & $\begin{array}{c}\begin{array}{c}\text { Cronbach's } \\
\text { alpha }\end{array} \\
\mathrm{N}=523\end{array}$ & $\begin{array}{c}\text { Test-retest } \\
\text { reliability ICC } \\
\mathrm{N}=81\end{array}$ & $\begin{array}{c}\text { Standardized } \\
\text { Factor Loading } \\
\text { (Final Model) } \\
\mathrm{N}=523\end{array}$ \\
\hline Coughing & 31,52 & 0.73 & 0.89 & $0.645-0.891$ \\
\hline Shortness of breath & $33-35$ & 0.82 & 0.93 & $0.573-0.964$ \\
\hline Fear of progression & 49,51 & 0.83 & 0.84 & $0.721-0.972$ \\
\hline Hair problems & 39,50 & $0 \cdot 78$ & 0.82 & $0.457-1.370$ \\
\hline $\begin{array}{l}\text { Surgery-related } \\
\text { symptoms }{ }^{2}\end{array}$ & $55-59$ & 0.86 & 0.97 & $0.685-0.813$ \\
\hline \multicolumn{5}{|l|}{ Side effects } \\
\hline $\begin{array}{l}\text { Symptom/side effect } \\
\text { burden score }\end{array}$ & $32,36-38,40-48,53,54$ & 0.71 & 0.89 & \\
\hline \multirow[t]{15}{*}{ Single items } & 32. Coughed up blood & & 0.49 & 0.886 \\
\hline & 36. Sore mouth or tongue & & $0 \cdot 76$ & 0.999 \\
\hline & 37. Problems swallowing & & 0.67 & 0.975 \\
\hline & 38. Tingling hands or feet & & $0 \cdot 70$ & 0.974 \\
\hline & 40. Chest pain & & 0.43 & 0.859 \\
\hline & 41. Pain in arm or shoulder & & $0 \cdot 74$ & 0.952 \\
\hline & 42. Pain in other parts of body & & 0.67 & 0.917 \\
\hline & 43. Allergic reactions & & 0.68 & 0.942 \\
\hline & 44. Burning or sore eyes & & 0.56 & 0.988 \\
\hline & 45. Dizziness & & 0.61 & 0.904 \\
\hline & 46. Splitting fingernails or toenails & & $0 \cdot 79$ & 0.967 \\
\hline & 47. Skin problems & & 0.75 & 0.949 \\
\hline & 48. Problems speaking & & 0.34 & 0.963 \\
\hline & 53. Decrease in physical capabilities & & 0.51 & 0.887 \\
\hline & 54. Weight loss problem & & 0.67 & 0.950 \\
\hline
\end{tabular}


Table 3 Convergent and discriminant validity

\begin{tabular}{|c|c|c|c|c|c|c|c|}
\hline & \multirow[b]{2}{*}{$\mathrm{N}$ items } & \multicolumn{3}{|c|}{$\begin{array}{c}\text { First assessment } \\
(n=523)\end{array}$} & \multicolumn{3}{|c|}{$\begin{array}{c}\text { Second assessment } \\
(n=195)\end{array}$} \\
\hline & & Convergent & Discriminant & Scaling error & Convergent & Discriminant & Scaling error \\
\hline Coughing & 2 & 0.58 to 0.58 & 0.04 to 0.49 & 0 & 0.65 to 0.65 & -0.02 to 0.42 & 0 \\
\hline Shortness of breath & 3 & 0.55 to 0.81 & 0.07 to 0.48 & 0 & 0.53 to 0.79 & -0.02 to 0.56 & 0 \\
\hline Fear of progression & 2 & 0.70 to 0.70 & 0.12 to 0.42 & 0 & 0.72 to 0.72 & 0.04 to 0.63 & 0 \\
\hline Hair problems & 2 & 0.65 to 0.65 & 0.04 to 0.29 & 0 & 0.69 to 0.69 & -0.02 to 0.34 & 0 \\
\hline Surgery-related symptoms & 5 & 0.63 to 0.75 & -0.15 to 0.50 & 0 & 0.57 to 0.79 & 0.06 to 0.59 & 0 \\
\hline Symptom/side effect burden score & 15 & 0.16 to 0.45 & -0.08 to 0.47 & 0 & 0.14 to 0.56 & -0.14 to 0.55 & 0 \\
\hline
\end{tabular}

Convergent validity: item-scale correlations with own scale corrected for overlap

Discriminant validity: item-scale correlations with other scales

Scaling error: number of definitive scaling errors, i.e. cases in which an item was significantly higher correlated with another scale 
Table 4 Responsiveness to change

\begin{tabular}{|c|c|c|c|c|c|}
\hline & $\mathbf{N}$ items & $\begin{array}{c}\text { Worse } \\
(n=50)\end{array}$ & $\begin{array}{l}\text { No change } \\
(\mathrm{n}=81)\end{array}$ & $\begin{array}{c}\text { Better } \\
(n=60)\end{array}$ & $\mathbf{p}$ \\
\hline \multicolumn{6}{|l|}{ QLQ-C30 } \\
\hline Physical functioning & 5 & $-12 \cdot 87$ & 2.04 & $3 \cdot 28$ & 0.001 \\
\hline Role functioning & 2 & $-16 \cdot 33$ & $2 \cdot 71$ & $9 \cdot 04$ & 0.001 \\
\hline Emotional functioning & 4 & $-4 \cdot 17$ & 3.44 & $11 \cdot 3$ & 0.001 \\
\hline Cognitive functioning & 2 & $-2 \cdot 00$ & $2 \cdot 71$ & $2 \cdot 22$ & 0.332 \\
\hline Social functioning & 2 & $-13 \cdot 33$ & $2 \cdot 74$ & $2 \cdot 22$ & 0.002 \\
\hline Global QoL & 2 & -11.05 & -0.63 & $10 \cdot 56$ & 0.001 \\
\hline Fatigue & 3 & $14 \cdot 44$ & $-4 \cdot 05$ & $-12 \cdot 05$ & 0.001 \\
\hline Nausea/vomiting & 2 & 2.00 & 0.62 & $-6 \cdot 21$ & 0.153 \\
\hline Pain & 2 & 5.67 & -1.23 & $-5 \cdot 28$ & 0.093 \\
\hline Dyspnea & 1 & $8 \cdot 67$ & $-6 \cdot 17$ & $-13 \cdot 56$ & 0.001 \\
\hline Sleep & 1 & $6 \cdot 67$ & -4.94 & $-11 \cdot 29$ & 0.009 \\
\hline Appetite loss & 1 & $12 \cdot 24$ & 0.41 & -12.99 & 0.001 \\
\hline Constipation & 1 & 9.03 & $-5 \cdot 35$ & -11.86 & 0.001 \\
\hline Diarrhea & 1 & 1.33 & 2.5 & -0.57 & 0.778 \\
\hline Financial difficulties & 1 & 5.44 & 0 & $1 \cdot 11$ & 0.476 \\
\hline \multicolumn{6}{|l|}{ QLQ-LC29 } \\
\hline Coughing & 2 & $-10 \cdot 00$ & $-3 \cdot 7$ & $-9 \cdot 04$ & 0.202 \\
\hline Shortness of breath & 3 & 9.98 & $-2 \cdot 4$ & $-6 \cdot 87$ & 0.001 \\
\hline Fear of progression & 2 & 8.84 & $-4 \cdot 12$ & -5.93 & 0.005 \\
\hline Hair problems & 2 & 8.00 & -0.62 & $7 \cdot 91$ & 0.032 \\
\hline Surgery-related symptoms & 5 & $-1 \cdot 48$ & $-2 \cdot 01$ & $-2 \cdot 67$ & 0.962 \\
\hline Symptom/side effect burden score & 15 & $6 \cdot 75$ & -0.92 & -2.53 & $<0.0001$ \\
\hline 32. Coughed up blood & 1 & 0.04 & -0.02 & -0.08 & 0.148 \\
\hline 36. Sore mouth or tongue & 1 & $0 \cdot 30$ & 0.04 & -0.03 & 0.019 \\
\hline 37. Problems swallowing & 1 & 0.26 & -0.04 & $-0 \cdot 14$ & 0.004 \\
\hline 38. Tingling hands or feet & 1 & 0.36 & -0.01 & $-0 \cdot 10$ & 0.003 \\
\hline 40. Chest pain & 1 & $0 \cdot 12$ & $0 \cdot 14$ & -0.20 & 0.034 \\
\hline 41. Pain in arm or shoulder & 1 & 0.02 & -0.02 & $-0 \cdot 14$ & 0.412 \\
\hline 42. Pain in other parts of body & 1 & 0.04 & $-0 \cdot 10$ & $-0 \cdot 19$ & 0.473 \\
\hline 43. Allergic reactions & 1 & -0.04 & -0.09 & 0.02 & 0.560 \\
\hline 44. Burning or sore eyes & 1 & $0 \cdot 20$ & 0.04 & -0.05 & 0.094 \\
\hline 45. Dizziness & 1 & 0.36 & 0.01 & -0.05 & 0.005 \\
\hline 46. Splitting fingernails or toenails & 1 & 0.06 & -0.06 & $0 \cdot 14$ & $0 \cdot 105$ \\
\hline 47. Skin problems & 1 & 0.31 & $-0 \cdot 14$ & $0 \cdot 17$ & 0.011 \\
\hline 48. Problems speaking & 1 & 0.29 & -0.07 & -0.07 & 0.005 \\
\hline 53. Decrease in physical capabilities & 1 & 0.45 & -0.14 & -0.22 & $<0.0001$ \\
\hline 54. Weight loss problem & 1 & 0.40 & 0.07 & $-0 \cdot 17$ & 0.001 \\
\hline
\end{tabular}

Means denote the changes between the second and first assessment; negative values in functioning (Physical to Global QoL) or positive values in symptoms (all other scores) indicate change for the worse. Means are presented for three groups of patients, according to their self-ratings on an anchor scale: worse, better or no change (compared to first assessment). 
Table 5 Known group differences: Karnofsky Performance Status

\begin{tabular}{|c|c|c|c|c|c|c|}
\hline & \multicolumn{3}{|c|}{ First assessment } & \multicolumn{3}{|c|}{ Second assessment } \\
\hline & $\begin{array}{c}\text { Karnofsky } \leq 70 \\
n=157\end{array}$ & $\begin{array}{c}\text { Karnofsky } \geq 80 \\
n=364\end{array}$ & $\mathrm{p}$ & $\begin{array}{c}\text { Karnofsky } \leq 70 \\
n=51\end{array}$ & $\begin{array}{c}\text { Karnofsky } \geq 80 \\
n=143\end{array}$ & $\mathrm{p}$ \\
\hline QLQ-C30 & & & & & & \\
\hline Physical functioning & $49 \cdot 98$ & $74 \cdot 74$ & $<0.0001$ & 49.93 & $74 \cdot 13$ & $<0.0001$ \\
\hline Role functioning & $39 \cdot 25$ & 67.91 & $<0.0001$ & $50 \cdot 00$ & $70 \cdot 28$ & $<0.0001$ \\
\hline Emotional functioning & $60 \cdot 22$ & 74.01 & $<0.0001$ & $73 \cdot 86$ & $75 \cdot 49$ & 0.669 \\
\hline Cognitive functioning & $74 \cdot 30$ & 83.38 & $<0.0001$ & $79 \cdot 41$ & 85.08 & 0.117 \\
\hline Social functioning & $48 \cdot 39$ & $73 \cdot 02$ & $<0.0001$ & $56 \cdot 21$ & $71 \cdot 36$ & 0.004 \\
\hline Global QoL & 44.52 & $63 \cdot 13$ & $<0.0001$ & $52 \cdot 61$ & $62 \cdot 32$ & 0.009 \\
\hline Fatigue & $61 \cdot 75$ & 38.63 & $<0.0001$ & $49 \cdot 46$ & $37 \cdot 30$ & 0.011 \\
\hline Nausea/vomiting & $19 \cdot 12$ & $10 \cdot 12$ & $<0.0001$ & $13 \cdot 73$ & 8.74 & $0 \cdot 124$ \\
\hline Pain & $45 \cdot 83$ & $22 \cdot 34$ & $<0.0001$ & 37.58 & $21 \cdot 41$ & 0.001 \\
\hline Dyspnea & $48 \cdot 60$ & $32 \cdot 78$ & $<0.0001$ & $35 \cdot 29$ & $29 \cdot 60$ & 0.233 \\
\hline Sleep & $40 \cdot 81$ & $29 \cdot 21$ & $<0.0001$ & 31.37 & $24 \cdot 24$ & 0.166 \\
\hline Appetite loss & 43.57 & 23.94 & $<0.0001$ & 37.91 & 23.54 & 0.007 \\
\hline Constipation & $38 \cdot 13$ & $20 \cdot 66$ & $<0.0001$ & 24.00 & $20 \cdot 28$ & 0.448 \\
\hline Diarrhea & $16 \cdot 34$ & $9 \cdot 81$ & 0.007 & $13 \cdot 33$ & $10 \cdot 02$ & 0.385 \\
\hline Financial difficulties & 29.87 & $19 \cdot 41$ & $<0.0001$ & $26 \cdot 67$ & $23 \cdot 40$ & 0.542 \\
\hline QLQ-LC29 & & & & & & \\
\hline Coughing & $37 \cdot 37$ & $27 \cdot 38$ & $<0.0001$ & 23.67 & $22 \cdot 11$ & 0.682 \\
\hline Shortness of breath & 44.01 & $27 \cdot 17$ & $<0.0001$ & 33.56 & $27 \cdot 74$ & 0.135 \\
\hline Fear of progression & $49 \cdot 47$ & $41 \cdot 12$ & 0.007 & $39 \cdot 33$ & 38.97 & 0.944 \\
\hline Hair problems & $20 \cdot 59$ & 21.66 & $0 \cdot 720$ & $20 \cdot 00$ & $22 \cdot 80$ & 0.572 \\
\hline Surgery-related symptoms & 35.98 & $22 \cdot 28$ & 0.001 & 38.96 & $15 \cdot 15$ & 0.007 \\
\hline Symptom/side effect burden score & $23 \cdot 37$ & 14.51 & $<0.0001$ & $19 \cdot 33$ & $14 \cdot 44$ & 0.003 \\
\hline 32. Coughed up blood & 1.23 & 1.09 & 0.005 & 1.20 & 1.05 & 0.075 \\
\hline 36. Sore mouth or tongue & 1.44 & 1.31 & 0.075 & 1.38 & 1.36 & 0.874 \\
\hline 37. Problems swallowing & 1.56 & 1.31 & 0.001 & 1.36 & 1.31 & 0.651 \\
\hline 38. Tingling hands or feet & 1.57 & 1.41 & 0.049 & 1.50 & 1.46 & 0.731 \\
\hline 40. Chest pain & 1.98 & 1.41 & $<0.0001$ & 1.72 & 1.40 & 0.007 \\
\hline 41. Pain in arm or shoulder & 1.88 & 1.43 & $<0.0001$ & 1.68 & 1.38 & 0.030 \\
\hline 42. Pain in other parts of body & $2 \cdot 17$ & 1.68 & $<0.0001$ & $2 \cdot 20$ & 1.66 & 0.001 \\
\hline 43. Allergic reactions & $1 \cdot 19$ & 1.17 & 0.737 & 1.14 & $1 \cdot 13$ & 0.911 \\
\hline 44. Burning or sore eyes & 1.35 & 1.30 & 0.393 & 1.24 & 1.32 & 0.444 \\
\hline 45. Dizziness & 1.76 & 1.41 & $<0.0001$ & 1.62 & 1.44 & 0.135 \\
\hline 46. Splitting fingernails or toenails & 1.29 & 1.29 & 0.975 & 1.28 & $1 \cdot 31$ & 0.825 \\
\hline 47. Skin problems & 1.83 & 1.71 & $0 \cdot 179$ & 1.90 & 1.79 & 0.483 \\
\hline 48. Problems speaking & 1.47 & $1 \cdot 18$ & $<0.0001$ & 1.28 & 1.21 & 0.427 \\
\hline 53. Decrease in physical capabilities & 2.96 & $2 \cdot 31$ & $<0.0001$ & $2 \cdot 60$ & 2.22 & 0.025 \\
\hline 54. Weight loss problem & $1 \cdot 84$ & 1.49 & $<0.0001$ & 1.60 & 1.47 & 0.355 \\
\hline
\end{tabular}

54. Weight loss problem

$1.49<0.0001$

effect items (items 32 to 54 ) range from 0 (=not at all) to 4 (= very much). 
Table 6 Patient characteristics $(N=523)$

\begin{tabular}{|c|c|}
\hline Median age (IQR)/years & $66(14)$ \\
\hline \multicolumn{2}{|l|}{ Gender } \\
\hline male & $315(60 \cdot 2 \%)$ \\
\hline female & $208(39.8 \%)$ \\
\hline \multicolumn{2}{|l|}{ Cohabitation status } \\
\hline Living with partner & $385(73 \cdot 6 \%)$ \\
\hline Living alone & $92(17.6 \%)$ \\
\hline Living with others & $43(8.2 \%)$ \\
\hline n.a. & $3(0.6 \%)$ \\
\hline \multicolumn{2}{|l|}{ Education } \\
\hline Less than compulsory school & $39(7.5 \%)$ \\
\hline Compulsory school & $219(41.9 \%)$ \\
\hline Post-compulsory school & $174(33.3 \%)$ \\
\hline University & $87(16 \cdot 6 \%)$ \\
\hline n.a. & $4(0.8 \%)$ \\
\hline \multicolumn{2}{|l|}{ Employment } \\
\hline Retired & $298(57.0 \%)$ \\
\hline Full time & $91(17.4 \%)$ \\
\hline Part time & $53(10 \cdot 1 \%)$ \\
\hline Homemaker & $23(4 \cdot 4 \%)$ \\
\hline Other & $54(10 \cdot 3 \%)$ \\
\hline n.a. & $4(0.8 \%)$ \\
\hline \multicolumn{2}{|l|}{ Smoking status } \\
\hline Non-smoker & $94(18.0 \%)$ \\
\hline Smoker & $112(21.4 \%)$ \\
\hline Ex-smoker & $316(60 \cdot 4 \%)$ \\
\hline n.a. & $1(0.2 \%)$ \\
\hline Median pack years (IQR) & $35(32.5)$ \\
\hline \multicolumn{2}{|l|}{ Disease stage } \\
\hline SCLC LD & $23(4.4 \%)$ \\
\hline SCLC ED & $58(11 \cdot 1 \%)$ \\
\hline NSCLC IA & $13(2 \cdot 5 \%)$ \\
\hline NSCLC IB & $13(2.5 \%)$ \\
\hline NSCLC IIA & $26(5.0 \%)$ \\
\hline NSCLC IIB & $25(4 \cdot 8 \%)$ \\
\hline NSCLC IIIA & $52(9.9 \%)$ \\
\hline NSCLC IIIB & $43(8.2 \%)$ \\
\hline NSCLC IV & $270(51.6 \%)$ \\
\hline \multicolumn{2}{|l|}{ Comorbidity (multiple entries permissible) } \\
\hline Renal & 20 \\
\hline Cardiac & 121 \\
\hline Respiratory & 101 \\
\hline Rheumatic & 26 \\
\hline Diabetes & 81 \\
\hline Liver & 6 \\
\hline Other & 181 \\
\hline \multicolumn{2}{|l|}{ Karnofsky Performance Status } \\
\hline n.a. & $2(0.4 \%)$ \\
\hline 30 & $1(0.2 \%)$ \\
\hline 40 & $7(1.3 \%)$ \\
\hline 50 & $24(4 \cdot 6 \%)$ \\
\hline 60 & $40(7.6 \%)$ \\
\hline 70 & $85(16 \cdot 3 \%)$ \\
\hline 80 & $181(34.6 \%)$ \\
\hline 90 & $143(27 \cdot 3 \%)$ \\
\hline 100 & $40(7.6 \%)$ \\
\hline Median (IQR) & $80(20)$ \\
\hline \multicolumn{2}{|l|}{ Study region } \\
\hline Enqlish-speaking country (U.K.) & $119(22.8 \%)$ \\
\hline Northern European countries (Germany, Norway, Belgium) & $174(33 \cdot 3 \%)$ \\
\hline Southern European countries (Cyprus, Israel, Italy, Spain, Greece) & $115(22.0 \%)$ \\
\hline Eastern European country (Poland) & $29(5 \cdot 5 \%)$ \\
\hline Non-European country (Jordan, Taiwan) & $86(16 \cdot 4 \%)$ \\
\hline \multicolumn{2}{|l|}{ Sample matrix (actual therapy at time of QoL assessment) } \\
\hline Surgery alone & $40(7 \cdot 6 \%)$ \\
\hline Surgery (late effects) & $38(7 \cdot 3 \%)$ \\
\hline Chemotherapy alone & $170(32.5 \%)$ \\
\hline Radiotherapy alone & $40(7.6 \%)$ \\
\hline Sequential radiochemotherapy & $16(3.1 \%)$ \\
\hline Concurrent radiochemotherapy & $42(8.0 \%)$ \\
\hline Targeted therapy alone & $70(13.4 \%)$ \\
\hline Targeted therapy in combination & $16(3 \cdot 1 \% 9)$ \\
\hline Immunotherapy & $91(17 \cdot 4 \%)$ \\
\hline
\end{tabular}


Curative

$164(31.4 \%)$

Palliative

$351(67.1 \%)$

n.a.

$8(1.5 \%)$ 